\title{
Diets Rich in Polyunsaturated Fatty Acids With Different Omega-6/Omega-3 Ratio Decrease Liver Content of Saturated Fatty Acids Across Generations of Wistar Rats
}

\author{
Simone Halfen ${ }^{1}$; Carolina Bespalhok Jacometo ${ }^{1}$; Patrícia Mattei ${ }^{1}$; Samanta Regine \\ Fenstenseifer $^{1}$; Luiz Francisco Machado Pfeifer ${ }^{2}$; Francisco Augusto Burkert Del Pino ${ }^{1}$; \\ Marco Aurélio Ziemann Santos ${ }^{3}$; Cláudio Martin Pereira de Pereira ${ }^{3}$; Eduardo Schmitt ${ }^{1}$; \\ Marcio Nunes Corrêa ${ }^{1} *$ \\ ${ }^{1}$ Universidade Federal de Pelotas, Centro de Pesquisa, Educação e Extensão Pecuária (NUPEEC), Pelotas, Rio \\ Grande do Sul, Brasil . . Empresa Brasileira de Pesquisa Agropecuária (Embrapa), Porto Velho, Rondônia, Brasil. ${ }^{3}$ \\ Universidade Federal de Pelotas - Instituto de Química e Geociências de Pelotas, Rio Grande do Sul, Brasil.
}

\begin{abstract}
Our study evaluated how the consumption of diets with low (LOW group - 0.4/1) or high (CON group - 13.6/1) omega-6/omega-3 ratio across generations (F1 and F2) can modulate liver fatty acid (FA) profile and blood biomarkers. Liver content of $\alpha$-linolenic acid was higher in animals always fed with LOW diet than animals that changed from CON to LOW diet, which by your time was higher than animals always fed with CON diet. Liver saturated FA concentration decreased in both groups from F1 to F2. In conclusion, both diets were efficient in decreasing the saturated FA liver content across generations, the LOW ratio diet was more effective in reducing blood triglycerides and non-esterified fatty acids, and there was a multigenerational effect of the LOW ratio diet, improving the FA profile even when the offspring start receiving the CON diet.
\end{abstract}

Key words: Alpha-linolenic acid, essential fatty acid, lipid, metabolism, nutrition, rats.

*Author for correspondence: marcio.nunescorrea@gmail.com 


\section{INTRODUCTION}

The liver has a central role in the metabolism, coordinating the synthesis and processing of fatty acids. Although the fat is an important nutrient and omega- 3 and omega- 6 are essential fatty acids, studies have highlighted the relation of unbalanced dietary fatty acids ratio and the risk factor associated with the development of metabolic syndromes (Jump 2011).

The essential fatty acids (EFAs) alpha-linolenic $(\alpha$-LNA; C18:3; n-3) and linoleic (LA; C18:2, n6 ) are the precursors of the very long chain polyunsaturated fatty acids (VLC-PUFA) eicosapentaenoic acid (EPA) and docosahexaenoic acid (DHA) of the n-3 family, and arachidonic acid and docosapentenoic acid (DPA) of the n-6 family (Herdt et al. 1988). These conversions between precursors and metabolites are dynamic in the body and catalyzed by a group of enzymes common for both families, basically the elongases and desaturases (Haggarty 2010). Through the activity of the enzymes cyclooxygenase and lipooxygenase, AA and EPA are converted to active eicosanoids (prostaglandins, leukotrienes and thromboxanes) that play important roles in cellular signaling and inflammation (Le et al. 2009).

The long chain polyunsaturated fatty acids (LCPUFAs) have important roles in the metabolism, during intrauterine life, being related to brain (Lenzi Almeida et al. 2011) and bone (Costa et al. 2012) development, and also during the postnatal period, being related to decreased occurrence of many diseases, as cardiovascular, atherosclerosis and inflammation (Stanley et al. 2007). During the last years great attention has been given to the importance of n-3 PUFAs in the regulation of lipid metabolism (Tai and Ding 2010), especially during the pathology of metabolic syndrome characterized by dyslipidemia like insulin resistance, obesity and hypertension (Lottenberg et al. 2012). Thus, the fatty acid profile of liver becomes an important tool in the analysis of interrelationships of lipid metabolism, which go from the intake of fatty acid in the diet, undergoes the reactions of elongation, desaturation and oxidation until the incorporation into tissue and metabolism modulation.
In order to achieve the effective benefits of the polyunsaturated fatty acids (PUFAs) consumption, the ratio of LA and $\alpha$-LNA must be considered, and studies in this field have been conducted for more than 10 years. The diets from Western society have excessive amounts of omega- 6 , with an estimated n-6/n-3 ratio of 15 $20 / 1$, so a lower ratio should be desirable in reducing the risk of the chronic diseases, varying from the ideal 1:1 to 5:1(Simopoulos 2002).

Considering the health benefits of PUFAs consumption, some studies demonstrate the nutrition effect during pregnancy, where the maternal nutrition has been one of the most important factors at the programming of division and redirection of nutrients to fetal growth and development (Gibson et al. 2011). It is already proved that maternal nutrition balance during pregnancy affect fetal body adiposity and its relation with offspring's risk of future disease (Blumfield et al. 2012). Plasma concentration of n-3 PUFAs are negatively associated with obesity (Micallef et al. 2009), however diets enriched with n-6 PUFAs was not effective in preventing excess liver lipid accumulation (Gaiva et al. 2003). Also, fatty acid nutrition during prenatal and early postnatal period may promote metabolic adaptations in the offspring related to fatty acid metabolism, and the epigenetic alterations could play an important role in this field (Niculescu et al. 2013).

Based on this, the aim of this study was to evaluate the hepatic fatty acid and metabolic profile of rats receiving diets with different $n$ 6/n-3 ratio across generations.

\section{MATERIAL AND METHODS}

\section{Animals, Diets and Experimental Procedures}

The experimental protocol was approved by the Animal Welfare Committee of Federal University of Pelotas (Rio Grande do Sul State, Brazil), under number 4382, and all procedures were conducted according to the guidelines of laboratory animal use in research. Male and female Wistar rats, 8 weeks old, were obtained from Central Vivarium/UFPel. Animals were housed (individually) in a temperature- (21$23^{\circ} \mathrm{C}$ ) and humidity-controlled $(60-70 \%)$ room with 12:12 h light-dark cycling (lights from 6 AM to 6 PM) and had free access to a pelleted diet and water. 
Diets were elaborated in accordance with AIN93G recommendations (AIN-93 for growth, pregnancy and lactation) (Reeves et al. 1993). Diets were isoproteic and isoenergetic having low or high $n-6 / n-3$ ratio. The low ratio diet
$($ LOW $=0.4 / 1)$, had flaxseed oil as energy source, while the high ratio diet $(\mathrm{CON}=13.6 / 1)$ had soybean oil as energy source (Table 1). Food intake was recorded daily.

Table 1. Ingredient composition of the experimental diets ( $\mathrm{g} / \mathrm{Kg} \mathrm{diet}$ )

\begin{tabular}{lcc}
\hline \multirow{2}{*}{ Ingredient } & \multicolumn{2}{c}{ AIN-93G $(\mathrm{g} / \mathrm{Kg}$ diet $)$} \\
\cline { 2 - 3 } Cornstarch & LOW & CON \\
Casein & 407.156 & 397.486 \\
Dextrinized cornstarch & 200.000 & 200.000 \\
Sucrose & 132.000 & 132.000 \\
Soybean oil & 100.000 & 100.000 \\
Flaxseed oil & 0.000 & 70.000 \\
Fiber & 60.330 & 0.000 \\
Mineral mix & 50.000 & 50.000 \\
Vitamin mix & 35.000 & 35.000 \\
L-Cystine & 10.000 & 10.000 \\
Choline bitratarte & 3.000 & 3.000 \\
Tert-Butylhydroquinone & 2.500 & 2.500 \\
\hline
\end{tabular}

The founding generation (G0) was composed of thirty-six females that were randomly assigned to one of two groups: (1) rats fed with a high n6/n-3 ratio (CON Group, n=18), and, (2) rats fed with low n-6/n-3 ratio (LOW Group, n=18). During the experimental period the males received only the CON diet. The animals were acclimatized to housing and diets for 30 days. Shortly thereafter, animals were mated in a male:female ratio of 1:3 for three days. The number and weight of offspring were recorded at birth, and the pups'development was accomplished by weekly weighing. From the G0 offspring, female progenies were sorted at weaning ( 21 days) to compose the $\mathrm{F} 1$ generation divided into three groups: (1) females from the CON group that continued receiving the diet with high $\mathrm{n}-6 / \mathrm{n}-3$ ratio $(\mathrm{CON} / \mathrm{CON}, \mathrm{n}=16)$; (2) females from LOW group that began to receive the high ratio diet (LOW/CON, $n=16$ ); and females from the LOW group that continued to receive the diet with lower $n-6 / n-3$ ratio (LOW/LOW, $\mathrm{n}=16$ ). Sixteen males were selected from the CON group. The animals were fed the diets for 60 days and then were mated as described above. From the F1 offspring, female progenies were selected at weaning ( 21 days) to compose the F2 generation, and the diet groups were maintained: (1) $\mathrm{CON} / \mathrm{CON} / \mathrm{CON}, \mathrm{n}=16$;
(2) LOW/CON/CON, $\mathrm{n}=16 ; \quad$ and LOW/LOW/LOW, $n=16$. To generate the F3 generation we followed the same approach as above for F2 generation. A schematic design of the groups can be seen at Figure 1. All females were evaluated for the pregnancy rate, number of pups per litter and average weight at birth.

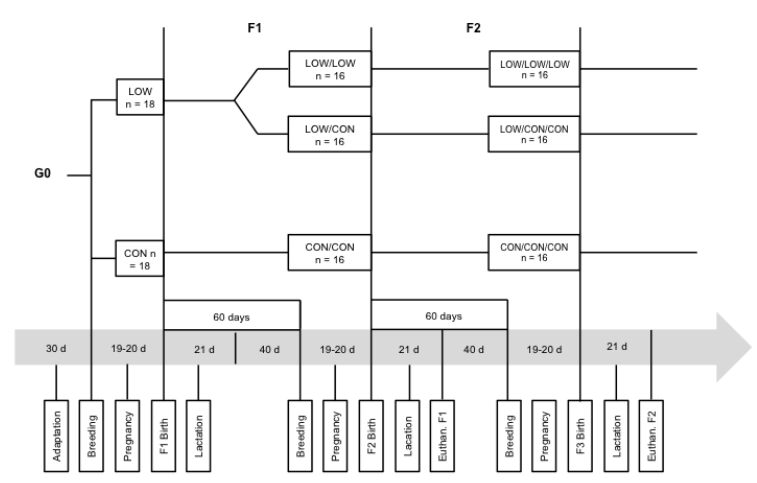

Figure 1 - Experimental design demonstrating the experimental groups in a timeline (G0, F1 and F2).

\section{Samples Collection}

Samples from the oils used to prepare the diets and a portion of the pelleted diet was collected monthly to evaluate the fatty acid (FA) profile during all the experimental period. 
In the F1 and F2 generations six females per group were fasted for $8 \mathrm{~h}$, anaesthetized and euthanized in the post lactation period (at weaning, 21 days postpartum) according to the protocol approved by the University Animal Care and Use Committee. Immediately after euthanasia the liver was collected, placed in cryotubes and submerged in liquid nitrogen at $196^{\circ} \mathrm{C}$, for posterior analysis of FA profile. Blood was collected by intracardiac puncture in clean and EDTA-FK containing tubes $(5 \mathrm{~mL}$ Vacuplast ${ }^{\circledR}$, Shandong, China). Upon collection, samples were centrifuged at $3000 \times \mathrm{x}$ for 15 min. Plasma was harvested and stored at $-80^{\circ} \mathrm{C}$ until analyzed. The samples were collected for measurement of plasma concentration of glucose, triglycerides and non-esterified fatty acids (NEFA).

\section{Biochemical Analysis}

The metabolites were analyzed using commercial biochemical assay kits, Glucose PAP Liquiform and Triglycerides (LabtestDiagnostica, Lagoa Santa, Brazil) in a visible light spectrophotometer (Biospectro SP
220, Curitiba, Brazil), and NEFA (Wako USA, Richmond, USA) using a microplate reader (Thermo Plate Reader, West Palm Beach, Florida, USA).

\section{Fatty Acid Profile}

All solvents and chemicals were of research grade and were obtained from Sigma-Aldrich (Saint Louis, Missouri, USA). Hepatic lipid extraction was performed as Bligh and Dyer Method (1959) and esterified as described by Hartman and Lago (1973). The analysis was performed on a Shimadzu 2010 Gas Chromatograph, with auto injection AOC-20i (Shimadzu, Kyoto, KYT, Japan) equipped with a $\mathrm{SP}^{\mathrm{TM}} 2560$ capyllary column (Supelco, SigmaAldrich, Saint Louis, Missouri, USA) with dimensions of $100 \mathrm{~m} \times 0,25 \mathrm{~mm}$ I.D. $(0,2 \mu \mathrm{m}$ film thickness). The used standard was the Frame Mix100m C4-C24 of Supelco, with injection of $1 \mu$ and split 100:1, for detection of until 36 FA. All results of fatty acids are presented as the percentage of total fatty acid (Table 2).

Table 2.Diets fattyacid profile (\% ofeachfattyacid) with high (CON) orlow (LOW) ratioof n-6/n-3.

\begin{tabular}{lcccc}
\hline & \multicolumn{4}{c}{ Diets } \\
\cline { 2 - 5 } n-6/n-3 ratio & LOW (flaxseedoil) & SEM* & CON (soybeanoil) & SEM $^{*}$ \\
\cline { 2 - 5 } Polyunsaturated & $0.410 / 1$ & $13.570 / 1$ & \\
Linoleic (C18:2) & 61.110 & 0.742 & 49.520 & 0.791 \\
a-linolenic (C18:3) & 17.750 & 1.180 & 45.990 & 0.540 \\
Eicosadienoic (C20:2) & 43.260 & 1.849 & 3.390 & 0.384 \\
Eicosatrienoic (C20:3) & $0.040 \mathrm{~b}$ & 0.005 & 0.070 & 0.013 \\
Eicosapentaenoic (C20:5) & 0.040 & 0.003 & 0.040 & 0.010 \\
Monounsaturated & 0.020 & 0.011 & 0.030 & 0.021 \\
Palmitoleic (C16:1) & 20.680 & 0.486 & 27.740 & 0.582 \\
Cis-10-Heptadecanoic (C17:1) & 0.180 & 0.003 & 0.160 & 0.004 \\
Oleic (C18:1) & 0.030 & 0.006 & 0.040 & 0.006 \\
Eicosenoic (C20:1) & 20.330 & 0.477 & 27.140 & 0.571 \\
Saturated & 0.140 & 0.022 & 0.410 & 0.061 \\
Caproic (C6:0) & 14.870 & 0.299 & 17.830 & 0.298 \\
Caprylic (C8:0) & 0.080 & 0.054 & 0.030 & 0.009 \\
Capric (C10:0) & 0.040 & 0.015 & 0.020 & 0.003 \\
Lauric (C12:0) & $0.060^{\mathrm{a}}$ & 0.002 & 0.050 & 0.002 \\
Myristic (C14:0) & 0.060 & 0.002 & 0.050 & 0.002 \\
Pentadecanoate (C15:0) & 0.370 & 0.006 & 0.330 & 0.010 \\
Palmitic (C16:0) & 0.080 & 0.001 & 0.060 & 0.001 \\
Heptadecanoic (C17:0) & 7.690 & 0.185 & 11.000 & 0.060 \\
& 0.100 & 0.001 & 0.110 & 0.001
\end{tabular}




\begin{tabular}{lllll} 
Stearic $(\mathrm{C} 18: 0)$ & 5.580 & 0.123 & 4.570 & 0.162 \\
Arachidic (C20:0) & 0.400 & 0.105 & 0.800 & 0.256 \\
Behenic (C22:0) & 0.240 & 0.017 & 0.560 & 0.012 \\
Tricosanoic (C23:0) & 0.050 & 0.002 & 0.060 & 0.007 \\
Lignoceric (C24:0) & 0.120 & 0.015 & 0.160 & 0.024 \\
Heneicosenoic (C21:0) & 0.010 & 0.002 & 0.030 & 0.003 \\
\hline
\end{tabular}

*SEM: standard error mean

\section{Statistical Analysis}

Results are presented as means \pm standard error of the mean. All the statistical analyses were performed using SAS 9.3 (SAS Institute Inc, Cary, Nort Carolina, USA). The pregnancy rate was compared among groups and generations by Chi-square. Diet consumption, diet and oil fatty acid profile, number and weight of pups were compared by One-way ANOVA and Tukey Test. For biochemical parameters and hepatic fatty acid profile the Mixed Models procedure was used, considering the effects of diet, generation, and its interaction. A value of $P<$ 0.05 was considered statistically significant.

\section{RESULTS}

The hepatic percentage of each fatty acid of the groups across generations can be found in the Supplemental File.

The $\alpha$-LNA mean content of liver samples of both generations were 10 fold higher $(P<0.01)$ in the LOW group (2.6\%) than the LOW/CON group $(0.24 \%)$ and 15 fold $(P<0.01)$ than the CON/CON group (0.17\%) (Fig. 2).

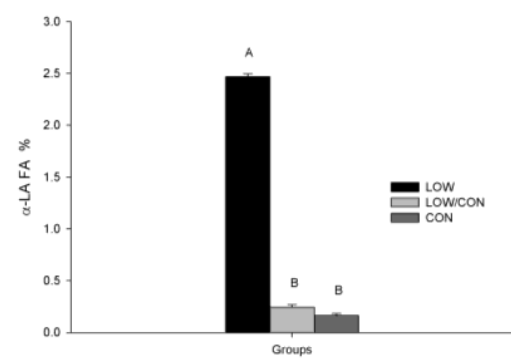

Figure 2 - Hepatic fatty acid (FA) percentage of alphalinolenic $(\alpha$-LA) FA fatty acid in the F1 and F2 generations in each group. Letters indicate differences between groups $(\mathrm{P}<0.05)$.

Disregarding groups, there was a decrease $(P=$ $0.01)$ from $F 1$ to $F 2$ in the total saturated FA composition, while the unsaturated FA content increased $(P=0.03)$ from $\mathrm{F} 1$ to $\mathrm{F} 2$ (Fig. 3).

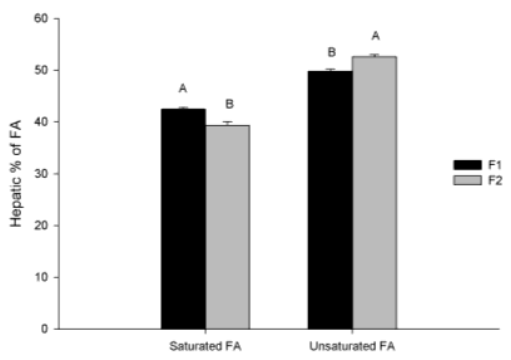

Figure 3 - Means of hepatic fatty acid percentage of saturated and unsaturated fatty acid (FA) in the F1 and F2 generations. Letters indicate differences of the same type of fatty acid between generations $(\mathrm{P}<0.05)$.

Analyzing the FA according to the number of double bounds, in a mean of both generation of each group, the LOW group had higher contents of FA with three double bounds than the LOW/CON $(P=0.01)$ and CON $(P<0.001)$ groups. The LOW group also had higher percentage $(P<0.01)$ of FA with five double bounds, and lower $(P<0.001)$ content of FA with two and four double bounds, comparing to LOW/CON and CON group. The CON group tended to higher $(P=0.08)$ content of monounsaturated FA (Fig. 4).

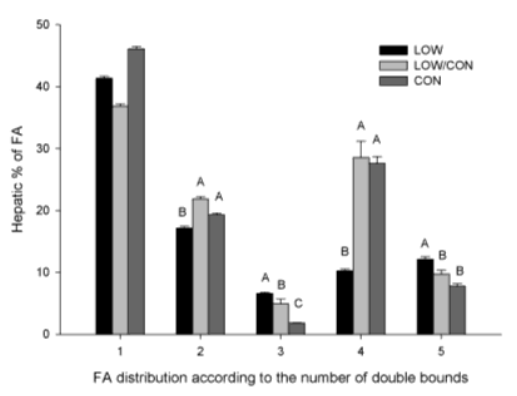

Figure 4 - Hepatic fatty acid (FA) percentage according to the number of unsaturation. Letters indicate differences between groups in the same class of fatty acid $(\mathrm{P}<0.05)$.

The blood biochemical analyses indicated that the fatty acid profile of the diet did not influence the homeostasis, as the glucose levels were similar in the groups and generations, and the animals were normoglycemic. The LOW diet decreased triglycerides in the F2 $(P=0.04)$ 
compared to the females that were receiving the CON diet. Between groups the only observed difference was in the F2 generation, where the CON/CON/CON group had higher NEFA concentration than LOW/LOW/LOW group $(P=$ $0.02)$, which in turn had higher concentration than the LOW/CON/CON group $(P=0.005)$.
We must highlight that although differences were observed between generations, the animals that received the LOW diet maintained a constant concentration of NEFA, while the CON group had an increase from $\mathrm{F} 1$ to $\mathrm{F} 2(P=0.005)$ (Table 3).

Table 3. Blood serum concentration ( $\mathrm{mmol} / \mathrm{L}$ ) of glucose, triacylglycerol (TAG) and non-esterified fatty acids (NEFA) in F1 and F2 generations in each group.

\begin{tabular}{cccccccc}
\hline Generation & Group & Glucose & SEM* & TAG & SEM* & NEFA & SEM* \\
\hline \multirow{2}{*}{ F1 } & LOW/LOW & 128.68 & 2.98 & 49.89 & 16.02 & 0.327 & 0.025 \\
& LOW/CON & 119.96 & 2.34 & 89.04 & 21.79 & 0.268 & 0.026 \\
& CON/CON & 130.85 & 10.98 & $60.41^{\mathrm{C}}$ & 11.78 & $0.258^{\mathrm{B}}$ & 0.035 \\
\hline \multirow{2}{*}{ F2 } & LOW/LOW/LOW & 141.054 & 4.51 & $57.93^{\mathrm{b}}$ & 8.56 & $0.329^{\mathrm{b}}$ & 0.035 \\
& LOW/CON/CON & 166.83 & 12.11 & $86.80^{\mathrm{ab}}$ & 11.60 & $0.220^{\mathrm{c}}$ & 0.007 \\
& CON/CON/CON & 144.18 & 4.43 & $109.25^{\text {Aa }}$ & 16.01 & $0.417^{\text {Aa }}$ & 0.034 \\
\hline
\end{tabular}

*SEM: standard error mean.

Capital letters indicate differences between generations in the same group $(\mathrm{P}<0.05)$.

Lowercase letters indicate differences between groups in the same generation $(P<0.05)$.

Diet consumption was recorded daily, and we could confirm that the diet FA profile (Table 2) did not affect the diet intake $(P>0.05)$. The mean daily intake for F1 (from its weaning until the F2 weaning) was $16.14 \pm 0.81 \mathrm{~g}$ for LOW/LOW group, $15.38 \pm 0.83 \mathrm{~g}$ for LOW/CON group and $16.90 \pm 0.74 \mathrm{~g}$ for $\mathrm{CON} / \mathrm{CON}$ group; and the F2 (from its weaning until the F3 weaning) was $18.02 \pm 0.99 \mathrm{~g}$ for LOW/LOW/LOW group, $19.67 \pm 0.68 \mathrm{~g}$ for
LOW/CON/CON group and $17.60 \pm 0.94 \mathrm{~g}$ for $\mathrm{CON} / \mathrm{CON} / \mathrm{CON}$ group.

The pregnancy rate was similar between the groups throughout the generations $(P>0.05)$, ranging from 62.5 to $87.5 \%$. There was no effect of the treatment on the number of pups per littler $(P>0.05)$, however in the $\mathrm{F} 1$ generation the pups mean weight at birth was greater in the LOW/LOW group than the CON/CON group $(P$ $=0.01)($ Table 4$)$.

Table 4. Pregnancy rate (\%), number of pups per female and mean pup birth weight (g) of the experimental groups along generations.

\begin{tabular}{ccccccc}
\hline \multirow{2}{*}{ Generation } & Group & $\begin{array}{c}\text { Pregnancy } \\
\text { rate }(\%)\end{array}$ & $\begin{array}{c}\text { Number of } \\
\text { pups/female }\end{array}$ & SEM* $^{*}$ & $\begin{array}{c}\text { Pup birth } \\
\text { weight (g) }\end{array}$ & SEM* \\
\hline \multirow{3}{*}{ F1 } & LOW/LOW & 62.50 & 8.60 & 1.36 & $7.33^{\mathrm{a}}$ & 0.21 \\
& LOW/CON & 62.50 & 7.28 & 1.11 & $6.70^{\mathrm{ab}}$ & 0.29 \\
& CON/CON & 81.25 & 9.56 & 1.07 & $6.55^{\mathrm{b}}$ & 0.13 \\
\multirow{3}{*}{ F2 } & LOW/LOW/LOW & 87.50 & 7.40 & 1.24 & 6.39 & 0.41 \\
& LOW/CON/CON & 62.50 & 10.00 & 1.10 & 7.27 & 0.19 \\
& CON/CON/CON & 75.00 & 10.43 & 1.56 & 6.88 & 0.41 \\
\hline
\end{tabular}

*SEM: standard error mean;

a-b:indicatessignificative differences $(P<0.05)$ between groups in the same generation.

\section{DISCUSSION}

Diet fatty acid composition is known to alter the fatty acid profile of stored and structural lipids in the body(Mohamed et al. 2002). Our results demonstrated a close link between dietary composition and tissue fatty acid profile across generations.

The higher hepatic $\alpha$-LNA concentration in the LOW group animals, in both generations reflects the direct effect of the diet, as the LOW diet had 
12 fold higher of $\alpha$-LNA than the CON diet (43.26\% and $3.39 \%$, respectively). However is important to note that the LOW/CON (F1) and $\mathrm{LOW} / \mathrm{CON} / \mathrm{CON}(\mathrm{F} 2)$ groups were receiving the CON, but the $\alpha$-LNA content was around $10 \%$ higher than the CON group, suggesting an effect of the G0 generation diet, as they received the LOW diet through intrauterine nutrition.

Across generations there was no cumulative effect in the incorporation of $\alpha$-LNA and LA FA, however the observed reduction of saturated and increase of unsaturated fatty acids along generations in the hepatic tissue is a promising result, as the unsaturated fatty acids rich diets can attenuate hepatic steatosis (Hanke et al. 2013; Janczyk et al. 2013). Kassem and colleagues(2012) also evaluated different diet ratios of n-3 and n-6 fatty acids to fed pregnant Sprague Dawley rats and found that diets with higher PUFAs n-6/n-3 ratios resulted in higher AA and lower DHA levels in plasma and linked this to the importance of DHA for fetal development, however when observing the hepatic fatty acid profile, no difference was observed in the content of $\alpha$-LNA and LA between the different ratios, concerning with our study. Connecting this data with molecular regulation, at gene expression level, results from our research group demonstrated, using this same experimental design, a down-regulation in the hepatic expression of the fatty acid synthase enzyme along generations (Jacometo et al. 2014). Also, recently there was evidenced that rats fed for 12 weeks with diets rich in poly- and monounsaturated

FA decreased fatty acid synthase protein levels in adipose tissue (Enns et al. 2014). This reduction in saturated fatty acids and decrease in endogenous fatty acid synthesis can be beneficial to the reduction of metabolic syndrome incidence.

Regarding the distribution of FA according to the number of double bounds, we can speculate the existence of a diet regulation in the desaturases enzymes activity and in the endogenous fatty acids synthesis pathway. A lipidomic approach confirmed the inhibition of de novo lipogenesis and alteration in the hepatic fatty acid profile via reduced desaturases activity induced by omega-3 fatty acids incorporated into hepatic phospholipids (Lamaziere et al. 2013).
As the diet fatty acid profile did not influence the consumption and animals' glycemia, indicates no effect at the basal metabolism, as reported in previous studies (Rice and Corwin 2002; Mellouk et al. 2012). Diets rich in n-3 and n-6 were effective in reduce plasma triglycerides levels (de Assis et al. 2012), and the reduction observed in the $\mathrm{F} 2$ of low $n-6 / n-3$ ratio, can also be an indicative of a reduction in the risk of developing metabolic syndromes (Poudyal et al. 2011).

Besides the triglycerides concentration on plasma, the decrease in blood NEFA concentration in the F2 generation on the group that always received the low $n-6 / n-3$ ratio, compared with the control diet, contribute to the evidences of the effectiveness of omega- 3 fatty acids in regulating the lipid metabolism. Similar results were found in a recent study with humans with diabetes mellitus that received n-3 supplementation, a significant reduction of NEFA was observed compared with the placebo group, and also improved insulin sensitivity (Farsi et al. 2014).

The lack of interaction between diet and pregnancy rate and number of pups along generations suggested that the ratio between $n-6$ and n-3 did not influence female fertility. DHA supplementation can slightly enhance the gestation length and promote an increase in the birth weight (Larque et al. 2012). Also,birth weight can be related to reduction in the body fat mass and consequently reduction in the obesity risks (Hauner et al. 2009), however the maternal supplementation seems to have no effect on offspring postnatal adipose tissue development (Much et al. 2013).

\section{CONCLUSIONS}

Our results showed that feeding animals with diets rich in PUFAs across generations modified the hepatic metabolism, reducing the concentration of saturated fatty acids while increasing the unsaturated fatty acids. The diet with lower $n-6 / n-3$ ratio provided reduced serum level of NEFA and triglycerides. Further there was a multigenerational effect of the LOW ratio diet, improving the FA profile even when the offspring start receiving the CON diet. Taken together previous researches and our results we can it is plausible to affirm that consuming diets 
rich in PUFAs exerts beneficial effects on health, improving the liver fatty acid profile and blood biochemical markers, leading to lower risk of developing metabolic syndrome related to lipid metabolism.

\section{ACKNOWLEDGEMENTS}

We thank the Central Vivarium of the University Federal of Pelotas for the provided structure and the all the collaborators of the Center of Research, Teaching and Extension in Animal Science (NUPEEC), by the support and assistance during the execution of project and laboratorial analysis. This research was funded by the PNPD Program (2278/2009) from the Coordination for the Improvement of Higher Level- or Education- Personnel (CAPES).

\section{REFERENCES}

Bligh EG,Dyer, WJ. A rapid method of total lipid extraction and purification. Can $J$ Biochem Physiol. 1959; 37: 911-917.

BlumfieldML, Hure AJ, Macdonald-Wicks LK, Smith R, SimpsonSJ, Giles WB, et al. Dietary balance during pregnancy is associated with fetal adiposity and fat distribution. Am J Clin Nut. 2012; 96, 1032-1041.

Costa CA, Carlos AS, Gonzalez GDEP, Reis RP, Ribeiro MDOS, Dos Santos ADE, et al. Diet containing low $n-6 / n-3$ polyunsaturated fatty acids ratio, provided by canola oil, alters body composition and bone quality in young rats. Eur $J$ Nutr. 2012; 51: 191-198.

De assis AM, Rech A, Longoni A, RottaLN, Denardin CC, Pasquali MA, et al. Omega3Polyunsaturated fatty acids prevent lipoperoxidation, modulate antioxidant enzymes, and reduce lipid content but do not alter glycogen metabolism in the livers of diabetic rats fed on a high fat thermolyzed diet. Mol Cell Biochem. 2012; 361: 151-160.

Enns JE, Hanke D, Park A, Zahradka P, Taylor CG. Diets high in monounsaturated and polyunsaturated fatty acids decrease fatty acid synthase protein levels in adipose tissue but do not alter other markers of adipose function and inflammation in diet-induced obese rats. Prostaglandins Leukot Essent Fatty Acids. 2014; 90: 77-84.

Farsi PF, Djazayery A, Eshraghian MR, Koohdani F, Saboor-Yaraghi AA, Derakhshanian $\mathrm{H}$, et al. Effects of supplementation with omega-3 on insulin sensitivity and non-esterified free fatty acid (NEFA) in type 2 diabetic patients. Arq Bras Endocrinol Metabol. 2014; 58: 335-340.

Gaiva MH, Couto RC, Oyama LM, CoutoGE, SilveiraVL, RibeiroEB, et al. Diets rich in polyunsaturated fatty acids: effect on hepatic metabolism in rats. Nutrition. 2003; 19: 144-149.

Gibson RA, Muhlhausler B, Makrides M. Conversion of linoleic acid and alpha-linolenic acid to longchain polyunsaturated fatty acids (LCPUFAs), with a focus on pregnancy, lactation and the first 2 years of life. Matern Child Nutr. 2011; $7:$ (2) 17 26.

Haggarty P. Fatty acid supply to the human fetus. Annu Rev Nutr. 2010; 30: 237-55.

Hanke D, Zahradka P, Mohankumar SK, Clark JL, Taylor CG. A diet high in alpha-linolenic acid and monounsaturated fatty acids attenuates hepatic steatosis and alters hepatic phospholipid fatty acid profile in diet-induced obese rats. Prostaglandins Leukot Essent Fatty Acids. 2013; 89: 391-401.

Hartman L, Lago RC. Rapid preparation of fatty acid methyl esters from lipids. Lab Pract. 1973; 22: 475-476.

Hauner H, Vollhardt C, Schneider KT, Zimmermann A, Schuster T, Amann-Gassner U. The impact of nutritional fatty acids during pregnancy and lactation on early human adipose tissue development. Rationale and design of the INFAT study. Ann Nutr Metab. 2009; 54: 97-103.

HerdtTH, Wensing T, Haagsman HP, Van Golde LM, BreukinkHJ. Hepatic triacylglycerol synthesis during a period of fatty liver development in sheep. J Anim Sci. 1988; 66: 1997-2013.

Jacometo CB, Schmitt E, Pfeifer LF, Schneider A, Bado F, da Rosa FT, et al. Linoleic and alphalinolenic fatty acid consumption over three generations exert cumulative regulation of hepatic expression of genes related to lipid metabolism. Genes Nutr. 2014; 9: 405-416.

Janczyk W, Socha P, Lebensztejn D, Wierzbicka A, Mazur A, Neuhoff-Murawska J, et al. Omega-3 fatty acids for treatment of non-alcoholic fatty liver disease: design and rationale of randomized controlled trial. BMC Pediatr. 2013; 13: 85-97.

Jump DB. Fatty acid regulation of hepatic lipid metabolism. Curr Opin Clin Nutr Metab Care. 2011; 14, 115-120.

Kassem AA, Abu Bakar MZ, Yong Meng G, Mustapham NM. Dietary (n-6 : n-3) fatty acids alter plasma and tissue fatty acid composition in pregnant Sprague Dawley rats. Sc World Jour. 2012; 85: 143-147.

Lamaziere A, Wolf C, Barbe U, Bausero P, Visioli F. Lipidomics of hepatic lipogenesis inhibition by omega 3 fatty acids. Prostaglandins Leukot Essent Fatty Acids. 2013; 88: 149-154. 
Larque E, Gil-Sanchez A, Prieto-Sanchez MT, Koletzko B. Omega 3 fatty acids, gestation and pregnancy outcomes. Br J Nutr. 2012; 107: (2), S77-84.

Le HD, Meisel JA, De Meijer VE, Gura KM, Puder M. The essentiality of arachidonic acid and docosahexaenoic acid. Prostaglandins Leukot Essent Fatty Acids. 2009; 81: 165-170.

Lenzi AKC, Teles BG, Guzman MA. Influence of omega-3 fatty acids from the flaxseed (Linum usitatissimum) on the brain development of newborn rats. Nutr Hosp. 2011; 26: 991-996.

Lottenberg AM, Afonso MDAS, Lavrador MS, Machado RM, NakandakareER. The role of dietary fatty acids in the pathology of metabolic syndrome. J Nutr Biochem. 2012; 23: 1027-1040.

Mellouk Z, Hachimi T, Louchami K, Hupkens E, Sener A, Yahia DA, et al. The metabolic syndrome of fructose-fed rats: effects of longchain polyunsaturated omega3 and omega6 fatty acids. II. Time course of changes in food intake, body weight, plasma glucose and insulin concentrations and insulin resistance. Int $\mathrm{J} \mathrm{Mol}$ Med. 2012; 29: 113-118.

Micallef M, Munro I, Phang M, Garg M. Plasma n-3 Polyunsaturated Fatty Acids are negatively associated with obesity. Br J Nutr. 2009; 102: 1370-1374.

MohamedAI, Hussein AS, BhathenaSJ, Hafez YS. The effect of dietary menhaden, olive, and coconut oil fed with three levels of vitamin $\mathrm{E}$ on plasma and liver lipids and plasma fatty acid composition in rats. J Nutr Biochem. 2002; 13: 435-441.

Much D, Brunner S, Vollhardt C, Schmid D, Sedlmeier EM, Bruderl M, et al. Effect of dietary intervention to reduce the $n-6 / n-3$ fatty acid ratio on maternal and fetal fatty acid profile and its relation to offspring growth and body composition at 1 year of age. Eur J Clin Nutr. 2013; 67: 282-288.
Niculescu MD, Lupu DS, Craciunescu CN. Perinatal manipulation of alpha-linolenic acid intake induces epigenetic changes in maternal and offspring livers. FASEB J. 2013; 27: 350-358.

Poudyal H, Panchal SK, Diwan V, Brown L. Omega3 fatty acids and metabolic syndrome: effects and emerging mechanisms of action. Prog Lipid Res. 2011; 50: 372-387.

Reeves PG, Nielsen FH, Fahey GC. AIN-93 purified diets for laboratory rodents: final report of the American Institute of Nutrition ad hoc writing committee on the reformulation of the AIN-76A rodent diet. J Nutr.1993; 123: 1939-1951.

RiceHB, Corwin, RL. Food intake in rats is unaffected by the profile of dietary essential fatty acids. Physiol Behav. 2002; 75: 611-619.

SimopoulosAP. The importance of the ratio of omega-6/omega-3 essential fatty acids. Biomed Pharmacother. 2002; 56: 365-379.

Stanley JC, ElsomRL, Calder PC, GriffinBA, HarrisWS, Jebb SA, et al. UK Food Standards Agency Workshop Report: the effects of the dietary n-6:n-3 fatty acid ratio on cardiovascular health. Br J Nutr. 2007; 98: 1305-1310.

Tai CC, Ding ST. N-3 polyunsaturated fatty acids regulate lipid metabolism through several inflammation mediators: mechanisms and implications for obesity prevention. $J$ Nutr Biochem. 2010; 21: 357-363.

Received: June 03, 2015; Accepted: September 02, 2015. 